\title{
Sonographic Diagnosis of Fetal Adrenal Hemorrhage Complicating a Vein of Galen Aneurysmal Malformation
}

\author{
Mehmet Serdar Kütük ${ }^{1}$, Selim Doğanay ${ }^{2}$, Ahmet Özdemir ${ }^{3}$, Süreyya Burcu Görkem², Adnan Öztürk \\ ${ }^{1}$ Department of Obstetrics and Gynecology, Erciyes University Faculty of Medicine, Kayseri, Turkey \\ ${ }^{2}$ Department of Radiology, Division of Pediatric Radiology, Erciyes University Faculty of Medicine, Kayseri, Turkey \\ ${ }^{3}$ Department of Pediatrics, Division of Neonatalogy, Erciyes University Faculty of Medicine, Kayseri, Turkey
}

Background: The vein of Galen aneurysmal malformation (VGAM) is a rare intracranial vascular malformation which causes end-organ ischemia or venous congestion due to heart failure. Adrenal hemorrhage associated with VGAM has not been reported in the literature. We present the imaging findings of a fetal VGAM with adrenal hemorrhage.

Case Report: A 26 year-old primigravida woman whose fetus with VGAM and mild cardiomegaly was scanned in the 34th week. On fetal ultrasound, a hyperechoic, well-circumscribed mass in the left suprarenal region was shown. Fetal and postnatal magnetic resonance imaging confirmed the diagnosis of adrenal hemorrhage. The baby died after delivery.

Conclusion: Adrenal hemorrhage can complicate VGAM in fetuses with severe heart failure. Evaluation of the adrenal gland in affected cases may contribute to the prenatal counseling, and postnatal management.

Keywords: Adrenal hemorrhage, fetal, ultrasound, vein of Galen aneurysmal malformation
The vein of Galen aneurysmal malformation (VGAM) is a rare intracranial vascular malformation which causes left-toright shunt and high-output cardiac failure and hydrocephalus, hemorrhage and fetal demise due to its mass effect (1). Endorgan ischemia and/or venous congestion may occur due to heart failure. Adrenal hemorrhage associated with VGAM has not been reported in the literature. In this report, we presented clinical features of a fetal VGAM which was complicated with adrenal hemorrhage with imaging findings.

\section{CASE PRESENTATION}

A 26 year-old primigravida female was referred to our center for intracranial cystic mass at 28 weeks of gestation. Her personal medical and obstetric history was unremarkable. Fetal ultrasound (US) (Voluson E8, GE Healthcare; Milwaukee, WI, USA) showed a 28 week-old, singleton fetus. Neurosonogram revealed a midline cystic lesion and dilated cavernous, transverse, and straight sinuses. On color Doppler examination, the cystic lesion had dilated and there were tortuous vascular structures which had high velocity arteriovenous shunt flow pattern (Figure 1). Fetal magnetic resonance imaging (MRI) (1.5T Aera; Siemens, Erlangen, Germany) confirmed the diagnosis of VGAM. Despite mild cardiomegaly, no valve regurgitation and pericardial or pleural effusion or ascites were observed. No additional anomaly was detected. At the $30^{\text {th }}$ week, cardiomegaly gradually increased with dilated carotid arteries demonstrated. At the $32^{\text {nd }}$ week, transabdominal fetal US revealed cardiomegaly with prominent tricuspid and pulmonary valve regurgitation, and mild pericardial effusion. Two weeks later ( $34^{\text {th }}$ week), hydrocephalus was also diagnosed. A hyperechoic homogenous mass which was $25 \times 25 \times 31 \mathrm{~mm}$ was detected at the left suprarenal region. The mass had a spherical shape with a central stripe as the character of a normal but enlarged adrenal gland. The ipsilateral kidney was separately demonstrated. No vascular structure was demonstrated in the mass on Doppler ultrasound (Figure 2a, b). Small amounts of anechoic fluid adjacent to the mass, extending to the left kidney, was also observed (Figure 2c). The fetal MRI confirmed the diagnosis of adrenal hemor-

Address for Correspondence: Dr. Süreyya Burcu Görkem, Department of Radiology, Division of Pediatric Radiology, Erciyes University Faculty of Medicine, Kayseri, Turkey Phone: +90 5309645409 e-mail: drburcugorkem@gmail.com

Received: 30.11.2014 Accepted: 10.07.2015 - DOI: 10.5152/balkanmedj.2015.151118

Available at www.balkanmedicaljournal.org 


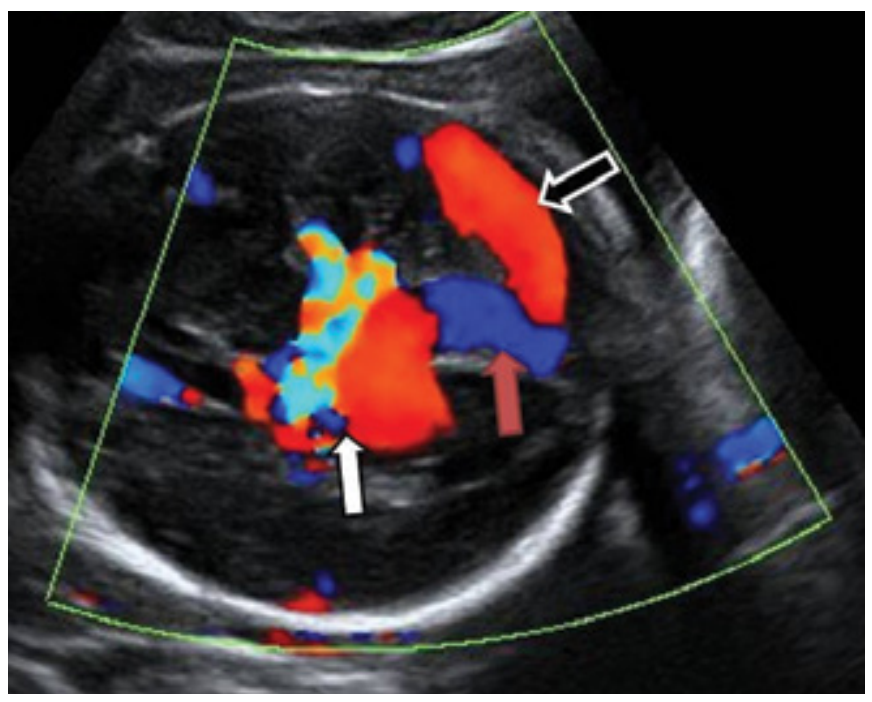

FIG. 1. On axial color Doppler image of the $30^{\text {th }}$ week fetus shows arteriovenous shunt flow pattern in the vein of Galen aneurysmal malformation (VGAM) (white arrow). Note the dilated straight and transverse sinuses (red and black arrow).

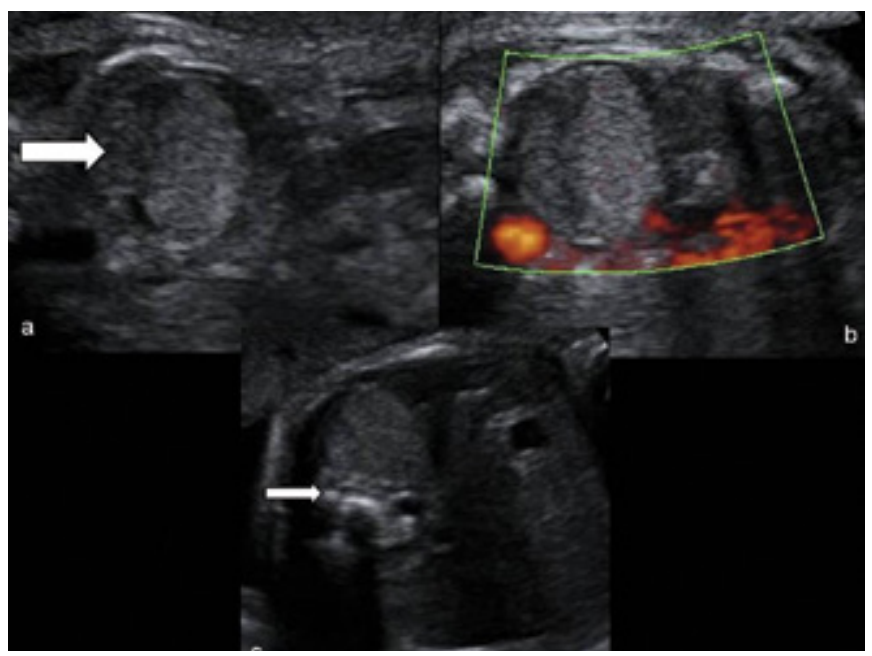

FIG. 2. a-c. Transabdominal fetal ultrasound at $34^{\text {th }}$ week. A central stripe in the well circumscribed left suprarenal mass demonstrates the normal adrenal gland (arrow) (a). On Doppler examination the mass has no vascularization (b). Transabdominal fetal ultrasound at $36^{\text {th }}$ week of left adrenal mass. A perirenal anechoic fluid indicates hemorrhage that ruptures from the capsule of the adrenal gland (arrow) (c).

rhage (Figure $3 \mathrm{a}, \mathrm{b}$ ). At the $36^{\text {th }}$ week, the dimensions of the mass were $34 \times 32 \times 36 \mathrm{~mm}$, and the echo texture did not change.

Maternal digoxin therapy was started, but fetal heart failure worsened progressively. An informed consent was taken from the patient and her husband. A cesarean section was scheduled at the $37^{\text {th }}$ week. The baby was male, $2270 \mathrm{gr} / 48 \mathrm{~cm}$, and had APGAR scores of 8 and 10 at one and five minutes, respectively. The tachypnea and tachycardia were prominent at birth. Despite positive inotropic support and diuretic therapy, severe heart failure, acidosis, and anuria ensued in the $12^{\text {th }}$ hour after birth. The neo-

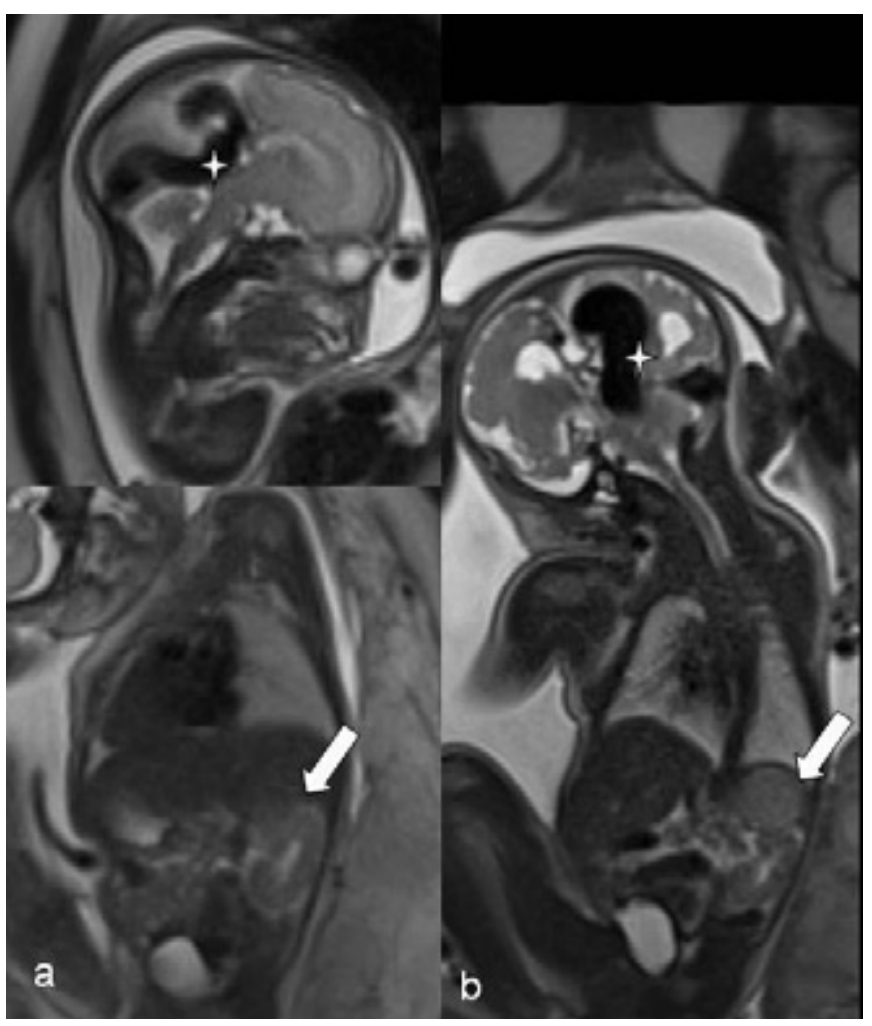

FIG. 3. a-b. On T2-HASTE fetal MRI ( $28^{\text {th }}$ week) there is hypointense vein of Galen aneurysmal malformation (VGAM) (star) which is demonstrated with normal left suprarenal area (arrow). The $34^{\text {th }}$ week fetal MRI shows that VGAM (star) has increased in size with hyperintense left adrenal hemorrhage on T2-HASTE coronal image (arrow).

nate did not respond to resuscitation and died at 18 hours postpartum. The family declined full autopsy, and the diagnosis was confirmed by postmortem MRI and multiple fine needle biopsies that gave coagulated blood and normal adrenal glandular histology.

\section{DISCUSSION}

In fetal and neonatal life, adrenal glands are vulnerable to hemorrhage because of their size (the fetal/neonatal size is about 20 times larger than the adult size) and high vascularity with supply from the inferior phrenic artery, abdominal aorta, and renal artery which predisposes hemorrhage/ischemia due to trauma or changes in blood pressure $(2,3)$. The incidence of acute adrenal hemorrhage in infants ranges from 0.2 to $3 \%$ (4). Risk factors include a history of anoxia or hypoxia during delivery, overweight for gestational age, prolonged labor, difficult extraction, sepsis, bleeding disorders, and renal vein thrombosis, or it may be idiopathic (4).

Fetal adrenal hemorrhage is a very rare entity and its diagnosis is challenging in the prenatal period. In the current ob- 
stetric practice, sonographic anatomical screening is performed between the $20^{\text {th }}$ and $23^{\text {rd }}$ week. Since adrenal hemorrhage almost always occurs in the third trimester, its prenatal diagnosis is usually not possible between the $20^{\text {th }}$ and $22^{\text {nd }}$ week (3). In our case, sonographic screening in the $20^{\text {th }}$ week was completely normal and the vein of VGAM was detected at $28^{\text {th }}$ week with cardiomegaly. The adrenal hemorrhage was detected in the $34^{\text {th }}$ week secondary to the worsening of heart failure. Although the right adrenal gland is more vulnerable to hemorrhage due to direct venous drainage to inferior vena cava, our case had left adrenal gland hemorrhage (5).

The main differential diagnosis of suprarenal masses includes neuroblastoma, extralobar pulmonary sequestration, congenital adrenal hyperplasia, partial multicystic dysplastic kidney, renal duplication, urinoma, gastric duplication cyst, and splenic cyst (6). Doppler study of the mass did not show any vascularity which excluded an adrenal solid mass e.g. neuroblastoma.

The sonographic appearance of adrenal hemorrhage varies during aging (3). Hemorrhage is often limited to the capsule of the adrenal gland. If it ruptures, blood may spread into the retroperitoneal space or peritoneal cavity, leading to scrotal hematoma and hydrocele (2). In our report, the hyperechoic mass strongly suggested acute hemorrhage. Additionally, the presence of free fluid along the adrenal gland and peritoneum was also a strong predictor of hemorrhage. In contrast to previous reports, we did not observe any resorption of the hemorrhage or rim-like calcifications which are the sonographic landmarks of bleeding. Continuing echogenicity of the hemorrhage indicates active bleeding $(3,7)$. The prognosis of adrenal hemorrhage is usually good unless it is complicated with other diseases. In fetuses with VGAM, the giant cerebral arteriovenous shunt causes a high output cardiac and due to the acute changes in the systemic pressure and/or failure vasoactive substances in circulation (e.g. Parkes Weber syndrome, or hereditary hemorrhagic telangiectasia) may cause hemorrhage secondary to venous congestion, such as the adrenal gland. Severe heart failure with adrenal hemorrhage in well vascularized organs in our case increased the risk of mortality. The high flow arteriovenous shunt in VGAM led to severe cardiac failure and death which needs emergency and intensive care in neonates. Postnatal embolization is beneficial only in babies without suprasystemic pulmonary hypertension (8-10).

According to the literature, no case of fetal adrenal hemorrhage associated with heart failure and hydrops with VGAM has been reported before. Thus, our case is the first.

In conclusion, VGAM can be seen in association with adrenal hemorrhage, and evaluation of the adrenal gland in affected cases may contribute to the prenatal counseling, and postnatal management. In order to ascertain the prognostic and clinical importance of adrenal hemorrhage in associated with VGAM, further studies with larger case numbers are required.

\section{Ethics Committee Approval: N/A.}

Informed Consent: Written informed consent was obtained from patient who participated in this study.

Peer-review: Externally peer-reviewed.

Author contributions: Concept - M.S.K., S.D.; Design - M.S.K., S.B.G.; Supervision - M.S.K.; Resource - M.S.K.; Materials A.Özdemir., S.D.; Data Collection \&/or Processing - S.D., M.S.D; Analysis \&/or Interpretation - M.S.K.; Literature Search - M.S.K., S.B.G.; Writing - S.B.G., M.S.K.; Critical Reviews - A.Özdemir, A.Öztürk.

Conflict of Interest: No conflict of interest was declared by the authors.

Financial Disclosure: The authors declared that this study has received no financial support.

\section{REFERENCES}

1. Deloison B, Chalouhi GE, Sonigo P, Zerah M, Millischer AE, Dumez Y, et al. Hidden mortality of prenatally diagnosed vein of Galen aneurysmal malformation: retrospective study and review of the literature. Ultrasound Obstet Gynecol 2012;40:652-8. [CrossRef]

2. Schrauder MG, Hammersen G, Siemer J, Goecke TW, Meurer $\mathrm{B}$, Hart N, et al. Fetal adrenal haemorrhage--two-dimensional and three-dimensional imaging. Fetal Diagn Ther 2008;23:72-5. [CrossRef]

3. Schwärzler P, Bernard JP, Senat MV, Ville Y. Prenatal diagnosis of fetal adrenal masses: differentiation between hemorrhage and solid tumor by color Doppler sonography. Ultrasound Obstet Gynecol 1999;13:351-5. [CrossRef]

4. Khuri FJ, Alton DJ, Hardy BE, Cook GT, Churchill BM. Adrenal hemorrhage in neonates: report of 5 cases and review of the literature. J Urol 1980;124:684-7.

5. Felc Z. Ultrasound in screening for neonatal adrenal hemorrhage. Am J Perinatol 1995;12:363-6. [CrossRef]

6. Maki E, Oh K, Rogers S, Sohaey R. Imaging and differential diagnosis of suprarenal masses in the fetus. $J$ Ultrasound Med 2014;33:895-904. [CrossRef]

7. Hsieh CC, Chao AS, Hsu JJ, Chang YL, Lo LM. Real-time and power Doppler imaging of fetal adrenal hemorrhage. Chang Gung Med J 2005;28:860-5.

8. Li AH, Armstrong D, terBrugge KG. Endovascular treatment of vein of Galen aneurysmal malformation: management strategy and 21-year experience in Toronto. J Neurosurg Pediatr 2011;7:3-10. [CrossRef]

9. Sammons V, Davidson A, Tu J, Stoodley MA. Endothelial cells in the context of brain arteriovenous malformations. J Clin Neurosci 2011;18:165-70. [CrossRef]

10. Revencu N, Boon LM, Mulliken JB, Enjolras O, Cordisco MR, Burrows PE, et al. Weber syndrome, vein of Galen aneurysmal malformation, and other fastflow vascular anomalies are caused by RASA1 mutations. Hum Mutat 2008;29:959-65. [CrossRef] 